\title{
Uromodulin identified as a potential therapeutic target
}

Novel findings have shown a link between the genetic susceptibility to salt-sensitive hypertension and chronic kidney disease (CKD) and the level of uromodulin expression. These findings, published by a team led by Olivier Devuyst and Luca Rampoldi in Nature Medicine, could offer up new clinical targets for the preservation of renal function and the treatment of hypertension.

Rampoldi explains the background: "This study stems from genetic evidence from multiple genome-wide association studies that common variants in the promoter of the UMOD gene give independent susceptibility for CKD and hypertension in many populations." The researchers hypothesized that the identified variants in the UMOD promoter could affect gene expression.

To test this hypothesis, "we used a translational approach based on cellular and mouse models, as well as human samples and wellcharacterized cohorts," explains Rampoldi. Using nephrectomy samples taken from patients, it was possible to measure the $U M O D$ transcript levels and establish that carriers of the highrisk $U M O D$ promoter variants also had high UMOD gene expression. This finding was confirmed in a large population-based cohort. Furthermore, in vitro studies showed that the common variants affected the transcriptional activity of the gene.

To establish the significance of this transcriptional activity, the genetic effect was recapitulated in mouse models. The mice expressed high levels of uromodulin and developed hypertension, which was caused by an abnormal reabsorption of sodium chloride by the kidney.

Using the transgenic mice, the researchers showed that the NKCC2 transporter (a cotransporter expressed with

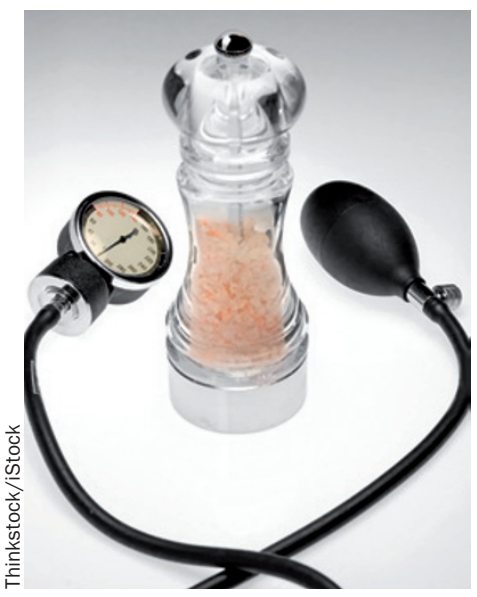

uromodulin in the thick ascending limb of the loop of Henle) was involved in this enhanced sodium chloride avidity. Hypertension was corrected in the mice by treating them with the loop diuretic furosemide. Importantly, among patients with hypertension, carriers of UMOD risk variants associated with high uromodulin expression showed a greater diuretic response and more marked drop in blood pressure in response to furosemide therapy.

The study also demonstrated that high levels of uromodulin expression are associated with renal damage, an effect that increases with age. "These findings are a major step forward as they clarify the biological effect of a genetic factor influencing the risk of hypertension and CKD," explains Devuyst.

Taken together, these results have explained the function of uromodulin-a protein that was first identified in urine 50 years ago. The next step will be to identify potential therapeutic targets for controlling blood pressure and preserving renal function.

Rebecca Kirk

Original article Trudu, M. et al. Common noncoding UMOD gene variants induce saltsensitive hypertension and kidney damage by increasing uromodulin expression. Nat. Med. doi:10.1038/nm.3384 